EPJ Web of Conferences 107, 03009 (2016)

DOI: $10.1051 /$ epjconf/201610703009

C) Owned by the authors, published by EDP Sciences, 2016

\title{
Manifestation of cluster effects in the structure of actinides
}

\author{
T.M. Shneidman ${ }^{1,2,3, a}$, G.G. Adamian ${ }^{1}$, N.V. Antonenko ${ }^{1}$, R.V. Jolos ${ }^{1}$, and Shan-Gui Zhou ${ }^{3,4}$ \\ ${ }^{1}$ Bogoliubov Laboratory of Theoretical Physics, JINR, Dubna, 141980 Russia \\ ${ }^{2}$ Kazan Federal University, Kazan 420008, Russia \\ ${ }^{3}$ State Key Laboratory of Theoretical Physics, Institute of Theoretical Physics, CAS, Beijing 100190, China \\ ${ }^{4}$ Center of Theoretical Nuclear Physics, National Laboratory of Heavy Ion Accelerator, Lanzhou 730000, China
}

\begin{abstract}
We developed a cluster model which allows to take into account both shape deformation parameters and cluster degrees of freedom. The model is based on the assumption that the wave function of nucleus can be treated as a superposition of a mononucleus and two-cluster configurations. The model is applied to describe the multiple negative-parity bands in deformed actinides. Special emphasis is made on the investigation of the recently measured positive parity $0_{2}^{+}$rotational band of reflection-asymmetric nature in ${ }^{240} \mathrm{Pu}$. The results suggest that this band might be understood as the one built on the lowest excited state in mass asymmetry degree of freedom.
\end{abstract}

\section{Introduction}

In the even-even isotopes of actinides and also in the heavy $\mathrm{Ba}$ and $\mathrm{Ce}$ isotopes the low-lying negative parity states are observed together with the usually presented collective positive-parity states combined into rotational or quasirotational ground-state bands. Formation of the positive-parity rotational or quasirotational bands is connected in general to the quadrupole collective motion, while the lowering of the negative-parity states is a signature of the presence of the reflection asymmetric collective mode.

There are several approaches to treat the collective motion related to the reflection asymmetric degrees of freedom (see the review [1] and references therein). In present work, we exploit the dinuclear system (DNS) concept developed in [2-4] that the collective oscillations of the nucleus in the mass asymmetry degrees of freedom lead to the formation with some probability of the cluster-type configurations. The contribution of the asymmetric cluster configurations to the intrinsic nuclear wave function leads to an appearance of a reflection-asymmetric deformation. The strength of this deformation is determined by the relative weight of the bicluster configurations. Although well established for light nuclei [5], the justification of the cluster approach to the description of the structure of heavy nuclei is cumbersome. However, there exist the evidences which support the idea of clustering. First of all, the nuclei whose spectra exhibit the strong reflectionasymmetric correlations are good alpha-emitters. Thus, there is a significant probability to form an $\alpha$-cluster in the surface region of the nucleus. It is also known from the Nillson-Strutinsky-type calculations for light nuclei that

\footnotetext{
a e-mail: shneyd@theor.jinr.ru
}

nuclear configurations corresponding to the minima of the potential energy contain particular symmetries which are related to certain cluster structures $[6,7]$. Several calculations performed for heavy nuclei [8-10] have shown that configurations with large quadrupole deformations and low-lying collective negative-parity states are strongly related to clustering. The energy of the cluster configurations with an alpha-particle are close to or even lower than the binding energy for these nuclei [2]. Therefore, the bicluster configurations with an $\alpha$-particle are expected to be important in the structure of actinides.

In [2-4], only the lowest negative parity bands $\left(K^{\pi}=0^{-}\right)$have been considered. At the same time there are experimental evidences which indicate on a presence of the collective states related to the reflection asymmetric modes and characterized by non zero values of $K$ (see, for example, [11]). Moreover, the recent investigations [12] have indicated that the reflection-asymmetric degrees of freedom can also contribute significantly to the structure of positive parity rotational band built upon excited $0_{2}^{+}$state which are observed in the excitation spectra of many actinides. To treat these collective excitations in the framework of our cluster approach we modify the model [2-4] to take into account the intrinsic excitations of clusters produced by the motion of the nucleus in mass-asymmetry degrees of freedom. In [13] we applied the modified model to treat the properties of ${ }^{220} \mathrm{Th}$. This is peculiar case of the nucleus characterized by the strong reflection-asymmetric deformation and relatively small quadrupole deformation. The aim of this work is to treat the situation when the nucleus has both strong quadrupole and strong reflectionasymmetric deformations. The model is applied for the description of the multiple-reflection asymmetric bands in heavy isotopes of actinides. 


\section{Model}

\subsection{The Hamiltonian}

Instead of parametrization of the nuclear shape in terms of multipole deformation parameters, we use the degrees of freedom related to the DNS or cluster system [14]. The DNS is understood as the system of two nuclei $\left(A_{1}, Z_{1}\right)$ and $\left(A_{2}, Z_{2}\right)$ kept together in touching configuration by the nucleus-nucleus potential. As a mononucleus, we consider a unified nuclear system with charge $Z_{1}+Z_{2}=Z$ and atomic number $A_{1}+A_{2}=A$. The degrees of freedom describing the collective excitations are related to the rotation of the DNS as a whole, to the relative motion of the DNS fragments, to the intrinsic excitations of the fragments, and to the transfer of nucleons between the DNS fragments. The latter process is described here with the massasymmetry $\xi=A_{2} / A$ and charge-asymmetry $\xi_{Z}=Z_{2} / Z$ coordinates. The values of $\xi=0$ or $\xi=1$ correspond to the mononucleus configurations $\left(A_{1}=A, A_{2}=0\right)$ or $\left(A_{1}=0, A_{2}=A\right)$, respectively.

The motion in $\xi$ destroys the reflection-symmetric shape of the nuclear system. The main idea of our approach is that the intrinsic nuclear wave function can be described as a superposition of the mononucleus and different dinuclear configurations, which are realized with certain probabilities. The mononucleus is taken to be quadrupole-deformed. The mass asymmetry $\xi$ is described as a continuous variable.

We presume that the main source of the reflection asymmetric deformation is the contribution of the DNS $|\alpha\rangle \times|(A-4, Z-2)\rangle$ with an alpha-particle as a light cluster. This idea is supported by the observation that the actinides are good $\alpha$-emitters. Thus, there is a significant probability to form an $\alpha$-cluster in the surface region of the nucleus. Moreover, our calculations show that the energy of the DNS with an alpha cluster $\left(\xi=\xi_{\alpha}\right)$, calculated as a sum of the binding energies of clusters and the energy of their interaction, are close to or even lower than the binding energy for these nuclei while the energies of configurations with light cluster heavier than alpha rapidly increase with $\xi$ [2]. Therefore, in order to treat the ground-state properties of actinides, one can consider the region of mass asymmetry in the vicinity of the mononucleus and alpha-cluster DNS configuration. The contribution of the configurations with $\xi>\xi_{\alpha}$ are negligibly small.

In the region of interest, the DNS, which are produced by the motion in mass-asymmetry, consist of a light spherical fragment $\left(A_{2}, Z_{2}\right)$ and the heavy deformed (with an axially-symmetric quadrupole deformation $\beta_{20}=\beta_{0}$ ) or spherical fragment $\left(A_{1}, Z_{1}\right)$. In addition to the motion in $\xi$, we consider the relative rotation of the DNS fragments, described by the angles $\Omega_{R}=\left(\theta_{R}, \phi_{R}\right)$, and rotation of the heavy fragment, described by the angles $\Omega_{h}=\left(\theta_{h}, \phi_{h}\right)$. Angles $\left(\theta_{R}, \phi_{R}\right)$ and $\left(\theta_{h}, \phi_{h}\right)$ are measured in the laboratory system (Fig. 1). One can introduce the plain angle $\epsilon$ between the symmetry axis of the heavy fragment and the vector of the relative distance $\mathbf{R}$.

The classical expression for the kinetic energy of the system with the chosen degrees of freedom is written as

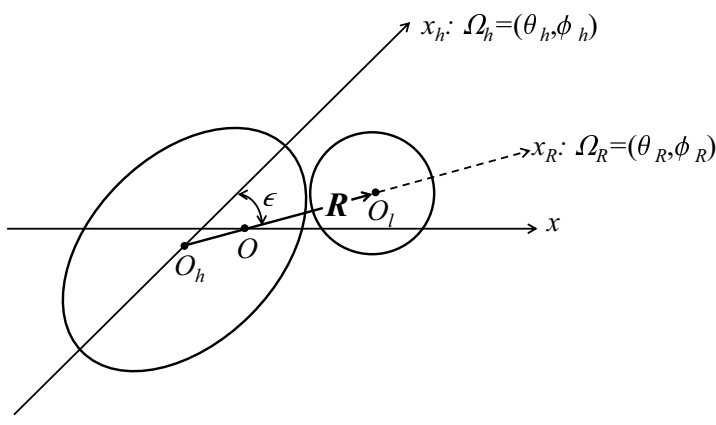

Figure 1. The schematic picture of the dinuclear system with the indicated degrees of freedom used. The orientation of the vector $\mathbf{R}$ connecting the centers of nuclei is defined by the angles $\Omega_{R}=\left(\theta_{R}, \theta_{R}\right)$ in the laboratory frame $O x_{R}$. The orientation of the intrinsic coordinate system $O_{h} x_{h}$ related to the quadrupole deformed heavy fragment is defined by the angles $\Omega_{h}=\left(\theta_{h}, \phi_{h}\right)$.

a sum of terms describing the motion in mass asymmetry, relative rotation of the DNS fragments, and rotation of the heavy fragment

$$
\begin{aligned}
T & =\frac{1}{2} B(\xi) \dot{\xi}^{2}+\frac{1}{2} \mu(\xi) R_{\mathrm{m}}^{2}(\xi)\left(\dot{\theta}_{R}^{2}+\theta_{R}^{2} \dot{\phi}_{R}^{2}\right) \\
& +\mathfrak{J}_{h}(\xi)\left(\dot{\theta}_{h}^{2}+\theta_{h}^{2} \dot{\phi}_{h}^{2}\right)
\end{aligned}
$$

Here, $\mu=m_{0} \frac{A_{1} A_{2}}{A} \approx m_{0} A \xi$ is the reduced mass of the nuclear system $(\xi \ll 1)$ where $m_{0}$ is the nucleon mass. The value of the relative distance $R=R_{\mathrm{m}}$ corresponds to the touching configuration for the certain value of $\xi$. The quantities $B(\xi)$ and $\mathfrak{J}_{h}(\xi)$ are the effective mass for the motion in mass asymmetry and the moment of inertia of the heavy fragment (or mononucleus). The method of calculation of $B(\xi)$ is described in Ref. [15]. In this work, however, we replace the effective mass parameter for the motion in mass asymmetry by its value $B\left(\xi_{0}\right)$ at average mass asymmetry $\xi_{0}, 0<\xi_{0}<\xi_{\alpha}$

$$
B(\xi)=B\left(\xi_{0}\right) .
$$

Because $\xi \ll 1$, in the quantization of the expression (1) we leave only the terms of the lowest order in $\xi$. As a result, the following expression is obtained for the quantum kinetic energy operator

$$
T=-\frac{\hbar^{2}}{2 B} \frac{1}{\xi} \frac{\partial}{\partial \xi} \xi \frac{\partial}{\partial \xi}+\frac{\hbar^{2}}{2 \mu(\xi) R_{\mathrm{m}}^{2}(\xi)} L_{R}^{2}+\frac{\hbar^{2}}{2 \mathfrak{J}_{h}(\xi)} L_{h}^{2},
$$

where the angular momentum operators have the form

$$
\begin{array}{r}
L_{i}^{2}=-\frac{1}{\sin \theta_{i}} \frac{\partial}{\partial \theta_{i}} \sin \theta_{i} \frac{\partial}{\partial \theta_{i}}-\frac{1}{\sin ^{2} \theta_{i}} \frac{\partial^{2}}{\partial \phi_{i}^{2}}, \\
(i=R, h) .
\end{array}
$$

The potential energy of the nuclear system with the mass asymmetry $\xi$ is written as

$$
U(\xi, \epsilon)=U_{0}(\xi)+U_{\epsilon}(\xi) \sin ^{2} \epsilon,
$$

where $\epsilon$ is related to $\Omega_{R}$ and $\Omega_{h}$ by the expression

$$
\sin ^{2} \epsilon=\frac{2}{3}\left(1-\frac{4 \pi}{\sqrt{5}}\left[Y_{2}\left(\Omega_{h}\right) \times Y_{2}\left(\Omega_{R}\right)\right]_{00}\right) .
$$


The dependence of the potential on $\epsilon$ is approximated by the second order expansion. In (5), the potential energy has a minimum which corresponds to the pole-to-pole orientation $(\epsilon=0)$.

Using (3) and (5) for the kinetic and potential energies, respectively, and, the expression (6) we obtain Hamiltonian of the model in the form

$$
\begin{aligned}
& H=H_{\xi}+H_{\text {rot }}+V_{\text {int }}\left(\xi, \Omega_{R}, \Omega_{h}\right), \\
& H_{\xi}=-\frac{\hbar^{2}}{2 B} \frac{1}{\xi} \frac{\partial}{\partial \xi} \xi \frac{\partial}{\partial \xi}+U_{0}(\xi)+\frac{2}{3} U_{\epsilon}(\xi), \\
& H_{\text {rot }}=\frac{\hbar^{2}}{2 \mu(\xi) R_{\mathrm{m}}^{2}(\xi)} L_{R}^{2}+\frac{\hbar^{2}}{2 \mathfrak{J}_{h}(\xi)} L_{h}^{2} \\
& V_{\mathrm{int}}=-\frac{8 \pi}{3 \sqrt{5}} U_{\epsilon}(\xi)\left\{Y_{2}\left(\Omega_{h}\right) \times Y_{2}\left(\Omega_{R}\right)\right\}_{00},
\end{aligned}
$$

where $H_{\xi}$ describes the motion in mass asymmetry, $H_{\text {rot }}$ describes the rotational motion of the heavy fragment and the relative rotation of the fragments, and $V_{\text {int }}$ describes the interaction between the rotational degrees of freedom.

The Hamiltonian (7) is diagonalized on the set of basis functions

$$
\Phi_{L M, \pi}^{l_{1}, l_{2}, n}=F_{n}(\xi)\left[Y_{l_{1}}\left(\Omega_{h}\right) \times Y_{l_{2}}\left(\Omega_{R}\right)\right]_{L M},
$$

where $n=0,1,2, \cdots, l_{1}=0,2,4, \cdots, l_{2}=0,1,2, \cdots$. Because the heavy fragment is assumed by the axially-symmetric quadrupole rotator, the quantum number $l_{1}$ can take only even values. Thus, the wave function does not change under the transformation: $\theta_{h} \rightarrow \pi-\theta_{h}, \phi_{h} \rightarrow \pi+\phi_{h}$. The angular part of the wave function (8) is given by the bipolar spherical harmonics which provide the proper transformation with respect to the rotation and space inversion. The parity of the state is then determined as $\pi=(-1)^{l_{2}}$.

The reduced transition probability for the transition from the initial state $|i\rangle$ to the final state $|f\rangle$ is calculated as

$$
B(E \lambda ; i \rightarrow f)=\frac{1}{2 I_{i}+1}\left|\left\langle f\left\|Q_{\lambda}\right\| i\right\rangle\right|^{2},
$$

where the multipole operator $Q_{\lambda \mu}$ is defined as

$$
\begin{aligned}
Q_{\lambda \mu} & =\sum_{\lambda_{1}, \lambda_{1}+\lambda_{2}=\lambda} \sqrt{\frac{4 \pi(2 \lambda+1) !}{\left(2 \lambda_{1}+1\right) !\left(2 \lambda_{2}+1\right) !}} \\
& \times q_{\lambda_{1} \lambda_{2}}(\xi)\left\{Y_{\lambda_{1}}\left(\Omega_{h}\right) \times Y_{\lambda_{2}}\left(\Omega_{R}\right)\right\}_{\lambda \mu},
\end{aligned}
$$

where

$$
\begin{aligned}
q_{\lambda_{1} \lambda_{2}} & =\sqrt{\frac{4 \pi}{2 \lambda_{1}+1}} \\
& \times\left[\left(\frac{A_{1}}{A}\right)^{\lambda_{2}} Q_{\lambda_{1}}^{(2)}+(-1)^{\lambda_{2}}\left(\frac{A_{2}}{A}\right)^{\lambda_{2}} Q_{\lambda_{1}}^{(1)}\right] R^{\lambda_{2}} .
\end{aligned}
$$

Here, $Q_{\lambda}^{(i)}(i=1,2)$ are the intrinsic multipole operators of the DNS fragments. Because we assume that the light fragment $\left(A_{2}, Z_{2}\right)$ is spherical and can not be excited in the considered energy range, the only nonzero moment for it is $Q_{0}^{(2)}=Z_{2} / \sqrt{4 \pi}$. The heavy fragment is quadrupole deformed and has nonzero moments: $Q_{0}^{(1)}=Z_{1} / \sqrt{4 \pi}$ and $Q_{2}^{(1)}=Q_{0} \approx \frac{3 Z_{1} R_{01}^{2}}{4 \pi} \beta_{0}$.
The explicit expressions for the dipole and quadrupole operators of the DNS are written in the form

$$
\begin{aligned}
Q_{1 \mu} & =\epsilon_{1} \sqrt{4 \pi} \frac{A_{1} Z_{2}-A_{2} Z_{1}}{A} R\left\{Y_{0}\left(\Omega_{h}\right) \times Y_{1}\left(\Omega_{R}\right)\right\}_{1 \mu}, \\
Q_{2 \mu} & =\epsilon_{2} \sqrt{4 \pi} \frac{A_{1}^{2} Z_{2}+A_{2}^{2} Z_{1}}{A^{2}} R^{2}\left\{Y_{0}\left(\Omega_{h}\right) \times Y_{2}\left(\Omega_{R}\right)\right\}_{2 \mu} \\
& +\frac{4 \pi}{\sqrt{5}} Q_{0}\left\{Y_{2}\left(\Omega_{h}\right) \times Y_{0}\left(\Omega_{R}\right)\right\}_{2 \mu},
\end{aligned}
$$

where $\epsilon_{i}(i=1,2)$ are the effective charges introduced for dipole and quadrupole transitions. The effective charge for $E 2$ transitions is set to be unity, $\epsilon_{2}=e$. For the $E 1$ transition, the effective charge is taken as $\epsilon_{1}=0.1 e$. The reason to introduce this renormalization is the following. As seen from (11), the results for the absolute values of the electric dipole moment are sensitive to the difference in $Z / A$ ratios of the fragments. In the present calculations we do not consider the charge asymmetry as an independent coordinate. Instead, we take the $Z / A$ ratio in the light fragment equal to 0.5 as in the alpha-particle. This consideration is, of course, oversimplified and requires the introduction of the effective charge.

\section{Results of calculation}

\subsection{Excitation spectra of actinides}

The results of the calculations of the energy spectra are presented in Fig. 2 together with available experimental data for ${ }^{230} \mathrm{Th},{ }^{232} \mathrm{U},{ }^{238} \mathrm{U}$, and ${ }^{240} \mathrm{Pu}$ nuclei. These are representatives of all considered nuclei. The experimental energies and spin and parity assignments are taken from [16]. One can see that the structure of the calculated spectra are very similar for all considered nuclei. The explanation that elucidate the peculiarities of the calculated spectra can be obtained by examination what basis function from the set given by (8) contributes most to the wave functions of the states of a given rotational band.

For the ground-state rotational band the angular part of the wave-function of the state with angular momentum $I$ is dominated by the function $\left[Y_{l_{1}=I}\left(\Omega_{h}\right) \times Y_{l_{2}=0}\left(\Omega_{0}\right)\right]_{I M}$. Since $\pi=(-1)^{l_{2}}$, the ground-state band contains only states of positive parity and even angular momentum. The wave functions of the lowest negative parity states have the dominant contribution from the component $\left[Y_{l_{1}=I}\left(\Omega_{h}\right) \times Y_{l_{2}=1}\left(\Omega_{0}\right)\right]_{(I+1) M}$. Such a solutions form a rotational band which contain the states of odd angular momentum and negative parity. This band can be interpreted as $K^{\pi}=0^{-}$band. One should note, however, that each eigenfunction of Hamiltonian (7) is superposition of states with different $K$ values, thus $K$ can be considered as a quantum number only approximately. The next lowest band of negative parity is formed from the states with main contribution of $\left[Y_{l_{1}=I}\left(\Omega_{h}\right) \times Y_{l_{2}=1}\left(\Omega_{0}\right)\right]_{(I-1) M}$ component for odd angular momentum and $\left[Y_{l_{1}=I}\left(\Omega_{h}\right) \times Y_{l_{2}=1}\left(\Omega_{0}\right)\right]_{I M}$ component for even angular momentum. This band can be approximately interpreted as $K^{\pi}=1^{-}$. In this case the angular momentum carried by the relative rotation is directed oppositely with the total angular momentum thus increasing the energy of this rotational band with respect to the 


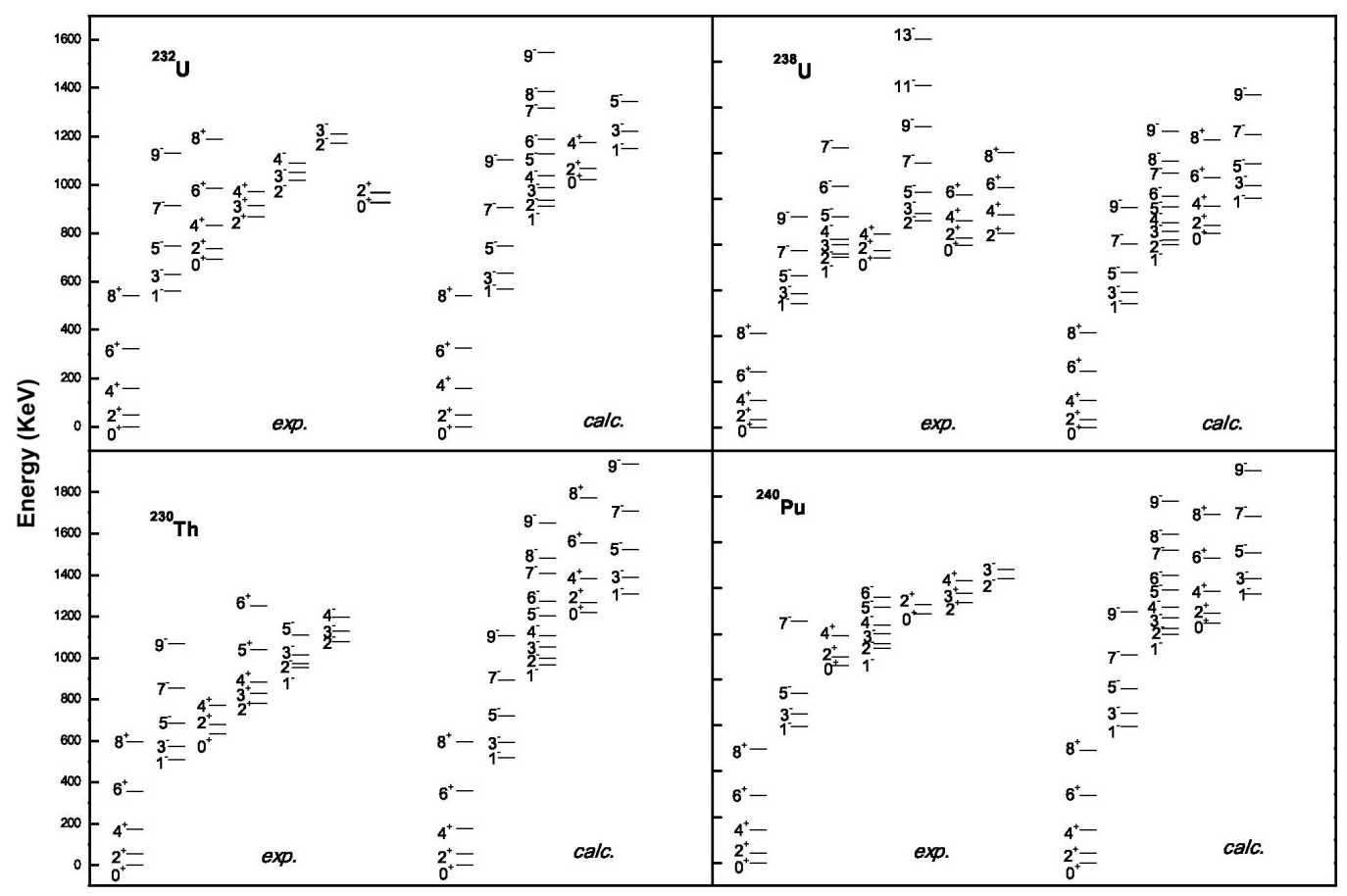

Figure 2. Low-lying states of ${ }^{230} \mathrm{Th},{ }^{232} \mathrm{U},{ }^{238} \mathrm{U},{ }^{240} \mathrm{Pu}$ obtained by the diagonalization of the Hamiltonian (7). The states are gathered into the rotational bands according to their structure in terms of basis functions (8). Calculated results are compared with low-energy experimental spectra of the corresponding nuclei. Experimental energies, spin and parity assignments are taken from [16].

states of $0^{-}$band. The first excited $0^{+}$state is obtained as a lowest excitation in the mass-asymmetry coordinate.

From Fig. 2 it is seen that the agreement between the calculated excitation spectra and corresponding experimental rotational bands are rather good for all presented nuclei. One can notice, however, that some of the bands measured experimentally, namely, $K^{\pi}=0^{+}, 2^{+}$, and $2^{-}$rotational bands, are absent in the calculated spectra. The reason for the lack of these bands is that in the present version of the model we have considered the heavy fragment with stable axially-symmetric quadrupole deformation. Thus, we may expect, that if we add into consideration the $\beta$ and $\gamma-$ vibrations of the heavy fragment, the calculated spectra of actinides will contain additional $0^{+}$and $2^{+}$rotational bands, which are build on the $\beta$ and $\gamma$-vibrational excitations, respectively. We can also expect, the appearance of additional low-lying $0^{-}$and $2^{-}$bands, as a negative parity doublets to the $\beta$ and $\gamma$-vibrational bands. Note that the alternative explanation of the appearance of the low-lying $2^{-}$bands in actinides is based on the concept of tetrahedral deformation $[17,18]$.

\subsection{Parity splitting}

An important quantity which determines the strength of the reflection-asymmetric deformation is the parity splitting [19] defined as the energy shift between the states of $0^{-}$and $0^{+}$rotational bands. Dependence of the experimental and calculated values of a parity splitting in the ground state and the first negative parity bands treated as a unified alternating parity band, on angular momentum is illustrated in Fig. 3. The parity splitting is

$$
S\left(I^{-}\right)=E\left(I^{-}\right)-\frac{(I+1) E_{(I-1)}^{+}+I E_{(I+1)}^{+}}{2 I+1},
$$

which provides zero value of parity splitting for the rotational band of a nucleus with rigid octupole deformation.

Having maximum value in the beginning of the band, the parity splitting tends to decrease with increase of angular momentum. In our model the value of parity splitting is determined by the relative weights of the cluster components and the mononucleus in the wave function. Due to the larger moment of inertia than that of the mononucleus, the weight of $\alpha$-particle DNS is increasing with angular momentum providing decrease of parity splitting.

\subsection{Dipole transitions in ${ }^{240} \mathrm{Pu}$}

With the wave functions obtained one can calculate the reduced transition matrix elements of the electric dipole and quadrupole transitions as defined in (11). In this contribution we present the results obtained in [20] for ${ }^{240} \mathrm{Pu}$. The dipole moments $D_{0}$ for the transitions between states of the ground state band, lowest negative parity band, and first excited $0^{+}$band are shown in Fig. 4. As seen, the dipole moment is an increasing function of angular momentum. Our model suggests the following explanation 

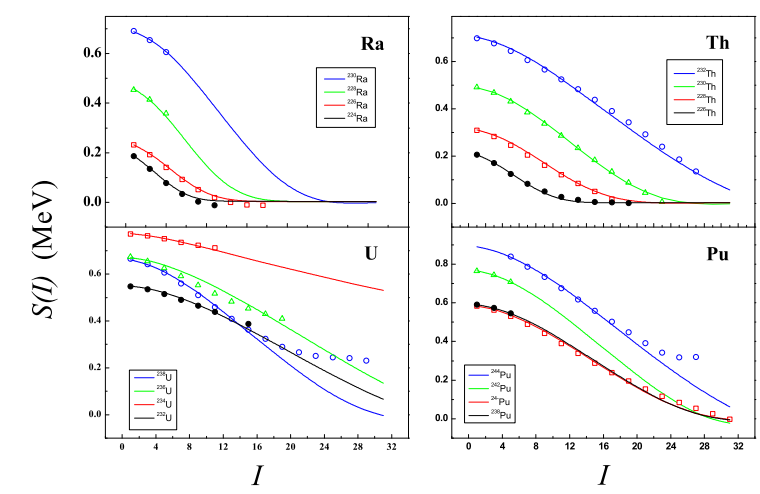

Figure 3. (Color online) Calculated (lines) and experimental (open circles of the same color) values of parity splitting (see (12)). Experimental values are taken from [16].

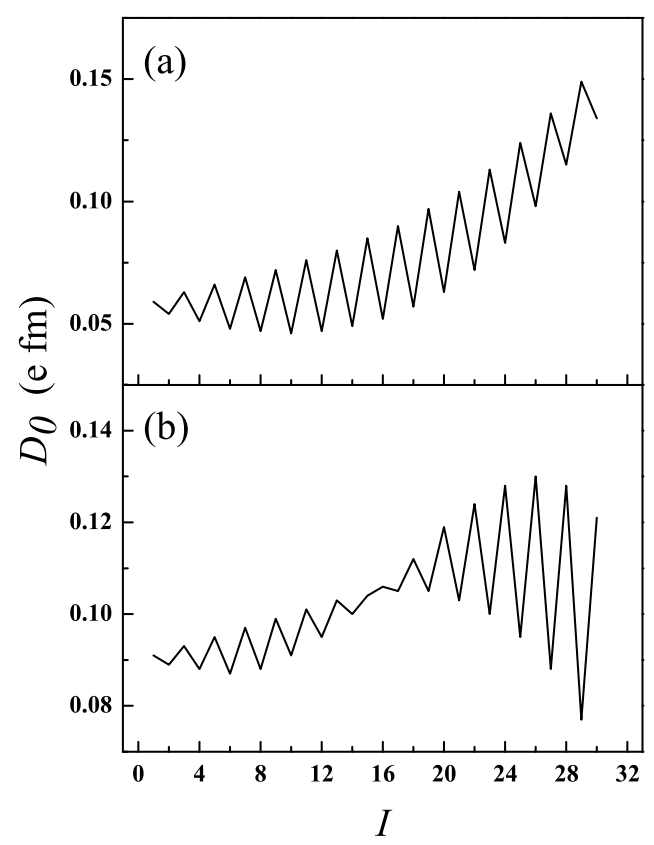

Figure 4. Transition dipole moment $D_{0}$ for the transitions between the first negative-parity band and the ground-state band (a), and between the first negative parity band and the second excited $0^{+}$band (b) as a function of the initial spin $I$.

of this effect. Due to different N/Z-ratios in the fragments, the dipole moment for the alpha-particle cluster system is large (for the pure alpha-particle DNS, $D_{0} \approx 4 e$ $\mathrm{fm})$. With increasing angular momentum the weight of the cluster configuration with an alpha-particle increases as well as the dipole moment. As seen, at $I_{i}<10$ the dipole moment for the transition between the ground-state band and the first negative-parity band lies in the range $D_{0} \approx(0.05-0.08) e \mathrm{fm}$. It increases with angular momentum and achieves the value $\sim 0.15 e \mathrm{fm}$ for $I_{i}>20$. This result is in good agreement with the experiment which suggests $D_{0} \sim 0.2 e$ fm for $I \geq 21$ [21].

As seen in Fig. 4, the dependence of the dipole moment on angular momentum demonstrates staggering behavior. The dipole moment for the transition from state

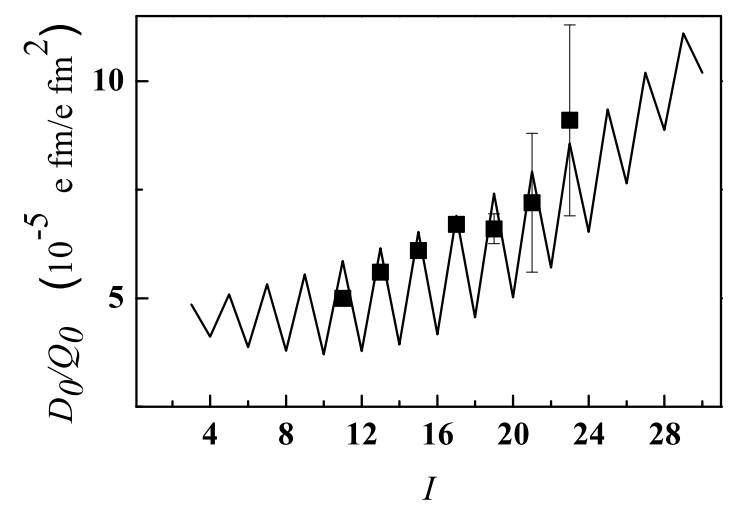

Figure 5. Ratio of transition dipole and quadrupole moments extracted from the $E 1$ and $E 2$ branching ratio $B\left(E 1, I^{-} \rightarrow(I-\right.$ $\left.1)^{+}\right) / B\left(E 2, I^{-} \rightarrow(I-2)^{-}\right)$as a function of $I$. The experimental results are extracted from Ref. [21].

$I$ of the ground-state band to state $(I+1)$ of the first negative-parity band is generally larger than for the transition to state $(I-1)$ of the same band. The reason of this staggering is the following. The basis functions (8), which mostly contribute to the state $I$ of the ground state band, have an angular part $\left[Y_{l_{1}=I}\left(\Omega_{h}\right) \times Y_{l_{2}=0}\left(\Omega_{R}\right)\right]_{I M}$. For the states $(I+1)$ and $(I-1)$ of the first negative parity band, the angular parts of the wave functions are mainly defined by $\left[Y_{l_{1}=I}\left(\Omega_{h}\right) \times Y_{l_{2}=1}\left(\Omega_{R}\right)\right]_{(I+1) M}$ and $\left[Y_{l_{1}=(I-2)}\left(\Omega_{h}\right) \times Y_{l_{2}=1}\left(\Omega_{R}\right)\right]_{(I-1) M}$, respectively. As follows from expression (11), the dipole operator cannot change the angular momentum of the heavy fragment. Thus, the transition $I \rightarrow(I-1)$ is hindered.

With increasing angular momentum the structure of the states becomes more complicated. For example, at $I \geq 15$ the contribution of the basis states $\left[Y_{l_{1}=(I-2)}\left(\Omega_{h}\right) \times Y_{l_{2}=2}\left(\Omega_{R}\right)\right]_{I M}$ to the wave functions of the ground-state band cannot be neglected. Because of this, the hindrance mechanism described loses its significance. As seen in Fig. 4(a), the staggering of $D_{0}$ decreases at large values of angular momentum. One can expect that with further increase of angular momentum the spectrum approaches its rotational limit and the staggering completely disappears.

The role of the structure of the wave functions is even more pronounced in the behavior of the dipole moment for the $E 1$ transitions from the excited $0^{+}$band to the first negative-parity band. This behavior is illustrated in Fig. 4 (b). The structure of the excited $0^{+}$band is more complicated than that of the ground-state band. Even in the beginning of the band the contribution of the basis function $\left[Y_{l_{1}=(I-2)}\left(\Omega_{h}\right) \times Y_{l_{2}=2}\left(\Omega_{R}\right)\right]_{I M}$ is important. Thus, for small angular momentum the staggering of $D_{0}$ is weak. However, with increasing angular momentum the staggering starts to grow and change its pattern. The reason for this is the contribution of the basis state $\left[Y_{l_{1}=(I-3)}\left(\Omega_{h}\right) \times Y_{l_{2}=3}\left(\Omega_{R}\right)\right]_{I M}$ to the wave function of the excited $0^{+}$band which becomes important at large angular momenta. 
Table 1. Calculated $r_{\text {calc. }}=B(E 1) / B(E 2)$ ratios are compared with the experimental values $\left(r_{\text {exp. }}\right)$ for the low-spin members of the excited $0^{+}$rotational band in ${ }^{240} \mathrm{Pu}$. The experimental values are taken from Ref. [12].

\begin{tabular}{ccccc}
\hline$I_{i}^{\pi}$ & $I_{f, E 1}^{\pi}$ & $I_{f, E 2}^{\pi}$ & $\begin{array}{c}r_{\text {exp. }} \\
\left(10^{-6} \mathrm{fm}^{-2}\right)\end{array}$ & $\begin{array}{c}r_{\text {calc. }} \\
\left(10^{-6} \mathrm{fm}^{-2}\right)\end{array}$ \\
\hline $0_{2}^{+}$ & $1_{1}^{-}$ & $2_{1}^{+}$ & $13.7(3)$ & 16.64 \\
$2_{2}^{+}$ & $1_{1}^{-}$ & $0_{1}^{+}$ & $99(15)$ & 66.92 \\
$2_{2}^{+}$ & $1_{1}^{-}$ & $2_{1}^{+}$ & $26(2)$ & 30.73 \\
$2_{2}^{+}$ & $1_{1}^{-}$ & $4_{1}^{+}$ & $5.9(3)$ & 8.14 \\
$2_{2}^{+}$ & $3_{1}^{-}$ & $0_{1}^{+}$ & $149(22)$ & 110.94 \\
$2_{2}^{+}$ & $3_{1}^{-}$ & $2_{1}^{+}$ & $39(2)$ & 50.99 \\
$2_{2}^{+}$ & $3_{1}^{-}$ & $4_{1}^{+}$ & $8.9(5)$ & 13.50 \\
$4_{2}^{+}$ & $3_{1}^{-}$ & $6_{1}^{+}$ & $4.4(11)$ & 6.97 \\
$4_{2}^{+}$ & $5_{1}^{-}$ & $6_{1}^{+}$ & $4.7(13)$ & 10.29 \\
\hline
\end{tabular}

For ${ }^{240} \mathrm{Pu}$, the calculated $D_{0} / Q_{0}$-ratio is presented in Fig. 5. One can see a good agreement with the available experimental data [21]. It is worth to note, that our model predict strong increase of $D_{0} / Q_{0}$-ratio at $I_{i} \geq 21$. It is presently hard to confirm this prediction because of the large experimental error-bars at large angular momentum.

Recently, the branching $B(E 1) / B(E 2)$-ratios were measured for the transitions from states of excited $0^{+}$band to states negative-parity band and to the ground-state band [12]. As seen in Table 1, the calculated results are in overall agreement with the experimental data.

\section{Conclusion}

We have suggested a cluster interpretation of the properties of the multiple negative parity bands in deformed even-even actinides. The collective motion related to the cluster degree of freedom leads to the admixture of the very asymmetric cluster configurations to the intrinsic nucleus wave function. To treat the reflection asymmetric modes with nonzero values of $K$, the rotational excitations of the heavy cluster is taken into account. The resulting energy spectrum consists of the ground state band, the excited $0^{+}$band and several negative parity rotational bands which can be approximately interpreted as $K^{\pi}=0^{-}$and $K^{\pi}=1^{-}$bands. The angular momentum dependence of the parity splitting is described. As an example, the electromagnetic transitions in ${ }^{240} \mathrm{Pu}$ were treated. The results of calculations support the cluster feature of the low-lying negative-parity states and some excited $0^{+}$bands.

\section{Acknowledgements}

This work was supported by the RFBR (Moscow) and NSFC (Beijing). T.M.S. acknowledges the support from the CAS Fellowship for the the Young International Scientists (2013Y1JA0003) and Russian Government Subsidy Program of the Competitive Growth of Kazan Federal University. S.G.Z. was partly supported by the 973 Program of China (Grant No. 2013CB834400), the NSF of China (Grants No. 11121403, No. 11120101005, and No. 11275248), and the Chinese Academy of Sciences (Grant No. KJCX2-EW-N01).

\section{References}

[1] P.A. Butler and W. Nazarewicz, Rev. Mod. Phys. 68, 349 (1996)

[2] T.M. Shneidman, G.G. Adamian, N.V. Antonenko, R.V. Jolos, and W. Scheid, Phys. Rev. C 67, 014313 (2003)

[3] G.G. Adamian, N.V. Antonenko, R.V. Jolos, and T.M. Shneidman, Phys. Rev. C 70, 064318 (2004)

[4] T.M. Shneidman, G.G. Adamian, N.V. Antonenko, and R.V. Jolos, Phys. Rev. C 74, 034316 (2006)

[5] K. Wildermuth and Y.C. Tang, Unified Theory of the Nucleus, Academic Press Inc (1977)

[6] W.D.M. Rae, Int. J. Mod. Phys. A 3, 1343 (1988)

[7] M. Freer and A.C. Merchant, J. Phys. G 23, 261 (1997)

[8] W. Nazarewicz, J.X. Saladin et al., Phys. Lett. B 322, 304 (1994)

[9] S. Åberg and L.-O. Jonsson, Z. Phys. A 349, 205 (1994)

[10] T.M. Shneidman, G.G. Adamian, N.V. Antonenko, S.P. Ivanova, and W. Scheid, Nucl. Phys. A 671, 119 (2000)

[11] W. Reviol et al., Phys. Rev. C 74, 044305 (2006)

[12] M. Spieker et al., Phys. Rev. C 88, 031303(R) (2013)

[13] T.M. Shneidman, G.G. Adamian, N.V. Antonenko, R.V. Jolos, and W. Scheid, Eur. Phys. J. A 47, 34 (2011)

[14] G.G. Adamian, N.V. Antonenko, and W. Scheid, Clusters in Nuclei, ed. Ch. Beck, Vol.2, Lecture Notes in Physics 848, (Springer-Verlag, Berlin, Heidelberg, 2012) p. 165.

[15] G.G. Adamian, N.V. Antonenko, and R.V. Jolos, Nucl. Phys. A 584, 205 (1995)

[16] http://www.nndc.bnl.gov/nndc/ensdf

[17] Y.S. Chen, Y. Sun, and Z.C. Gao, Phys. Rev. C 77, 061305(R) (2008)

[18] J. Zhao, B.-N. Lu, E.-G. Zhao, and S.-G. Zhou, Phys. Rev. C 86, 057304 (2012)

[19] R.V. Jolos and P. von Brentano, Phys. Rev. C 49, 2301(R) (1994)

[20] T.M. Shneidman, G.G. Adamian, N.V. Antonenko, R.V. Jolos, S.-G. Zhou, Phys. Rev. C 92, 034302 (2015)

[21] I. Wiedenhover et al., Phys. Rev. Let. 83, 2143 (1999) 